\title{
MVDC Supply Technologies for Marine Electrical Distribution Systems
}

\author{
Uzair Javaid, Francisco D. Freijedo, Drazen Dujic, and Wim van der Merwe
}

\begin{abstract}
The increase in the popularity of the medium voltage de (MVDC) electrical distribution, as a possible evolution of the medium voltage ac (MVAC) electrical distribution for the ship onboard power systems, arises a need for a comparative evaluation and demonstration of feasible technologies for the MVDC supplies. For designing the MVDC supplies in the range from 5-35 kV, different available technologies can be considered for the designs and have a direct influence on the overall system performance. In this paper, different technologies for prime movers, electrical generators and rectifiers are discussed in terms of feasibility for the MVDC supplies. Different supply configurations can be envisioned from these based on the commercial availability, quality of supply, efficiency, dynamic performance and volume. Multi-phase multipulse supply configurations are identified and proposed for the marine MVDC systems. Combination of multi-phase generators and multi-pulse rectifiers offer reliable, simple and fault tolerant solution with acceptable dynamics. To explore and highlight these benefits, a six-pulse rectifier sub-module is designed and analysed in two arrangements for multi-pulse configurations, namely parallel and series. It has been shown that with appropriate selection of semiconductor devices, coupled with properly selected fast fuses, excellent fault current (thermal) withstand capabilities can be achieved.
\end{abstract}

Index Terms-Fault tolerance, multi-phase generation, multipulse rectifiers, MVDC electrical distribution, MVDC supplies.

\section{INTRODUCTION}

$\mathrm{S}_{\mathrm{b}}^{\mathrm{N}}$ $\mathrm{NCE}$ the last decade, both academia and industry have been exploring the possibilities of dc distribution for the ship on-board electrical distribution system [1]-[3]. This has lead to the development of a low voltage dc (LVDC) electrical distribution system $(1 \mathrm{kV})$ for offshore support vessels by $\mathrm{ABB}$, covering power requirements of up to $20 \mathrm{MW}$, with reported fuel savings reaching 20\% [2]. Another LVDC system is developed by Siemens named BlueDrive PlusC [4]. These systems demonstrate the benefits of the dc electrical

Manuscript received November 22, 2017. This work is continuation of the conference paper: U. Javaid, F. D. Freijedo, D. Dujic and W. van der Merwe, "Marine MVDC Multi-Phase Multi-Pulse Supply," 43rd Annual Conference of the IEEE Industrial Electronics Society(IECON), Beijing, Oct. 29-Nov. 1, 2017, 6807-6812. (Corresponding author: Uzair Javaid).

U. Javaid, F. D. Freijedo, and D. Dujic are with Power Electronics Laboratory, École Polytechnique Fédérale de Lausanne(EPFL), Station 11, CH-1015 Lausanne, Switzerland (e-mail: uzair.javaid@epfl.ch; francisco.freijedo@epfl. ch; drazen.dujic@epfl.ch).

W. van der Merwe is with Medium Voltage Drives, ABB Switzerland Ltd, Austrasse, CH-5300, Turgi, Switzerland (e-mail: wim.van-der-merwe@ ch.abb.com).

Digital Object Identifier 10.24295/CPSSTPEA.2018.00007 distribution in ships such as lower fuel usage, increased energy density of the system, smaller footprint of the installed equipment, and flexibility in overall ship design. Higher power requirements (more than $20 \mathrm{MW}$ ) in larger ships, where the state-of-the-art is the medium voltage ac (MVAC) electrical distribution [5], require migration to medium voltage dc (MVDC) electrical distribution system to achieve the same benefits as shown by LVDC systems.

For the implementation of the MVDC electrical distribution system on ships, one of the key areas for research and development is the on-board electricity generation. In the present systems, i.e., MVAC electrical distribution systems, fixed speed operation of diesel engines is required to maintain ac frequency and the generators are operated in a synchronized manner. The move to the MVDC electrical distribution system removes these synchronization requirements, as the generators are interfaced to the rectifiers and the diesel engines could be operated at variable speeds, achieving optimum fuel consumption and efficient system operation. Possible technologies, for the MVDC supply, have been discussed in literature, highlighting the possible prime movers, generators, rectifiers and energy storage that could be used [6]-[12]. In [6], 7\% fuel savings is predicted for the MVDC electrical distribution, where two similar ship onboard systems are compared with one having MVAC and the other with MVDC electrical distribution. In addition to the fuel savings, this opens up opportunities to use high speed prime movers and generators, resulting in higher energy density and smaller footprint [3]. In [7], this prospect is further investigated considering generator technologies starting from the existing low- to high-speed generators and extending to the possible ultra-high speed generators in the future. The possibility of interfacing multi-phase generators with multi-pulse rectifiers, connected in series, is considered in [8], [9], whereas, their impact on the stability of the distribution system is evaluated in [10]. The impact of charging of energy storage on dc-side voltages is discussed in [11], [12].

Another important aspect of the MVDC electrical distribution system is its fault tolerant characteristics. As dc breakers are not readily commercially available for MVDC applications, different works have been presented on possible ways of limiting or blocking the fault current in a very short time, i.e., 4-10 ms [13]-[17]. Different hybrid fault clearing circuits are proposed in [13], [14], but they usually result in high losses [16]. Fault clearing topology for active rectifiers that can isolate the fault current, is presented in [15], while current fold-back principle is proposed for thyristor rectifiers for MVDC marine applications, in [16]. The LVDC electrical distribution systems for ships de- 
ploy multiple strategies to handle high fault currents and ensure high system availability. These strategies include, e.g., generators with high sub-transient reactance $\left(\mathrm{X}_{g}{ }^{\prime \prime}\right)$, overload detection equipment on generators, specially developed fault tolerant busties, fuses, isolator switches, fault current blockage by power converters and short circuit withstand capability of power converters [2], [17].

This paper provides an overview of the feasible MVDC supply technologies, and in particular highlights the advantages of multi-phase multi-pulse supplies. Possible multi-phase multi-pulse supplies are proposed for the MVDC distribution and design of a rectifier sub-module is provided that can be arranged in parallel and series configurations to generate different MVDC distribution voltages. As DC breakers are unavailable, surge current and fault handling capabilities of the proposed rectifiers are analyzed and fast fuses are considered to remove high fault currents. Furthermore, the paper is organized as follows: Section II provides brief qualitative analysis based on commercially available prime movers, generators and rectifiers, commonly used in MVAC system and thus considered ready for the MVDC systems. Possible MVDC supply configurations, predominantly considering various configuration and topologies of rectifiers are presented in Section III, from where system level advantages of multi-phase multi-pulse rectifier configurations are derived. Section IV presents the design of practical MVDC supplies and an analysis is provided considering the surge current and thermal withstand capabilities of the proposed rectifier topologies. Finally, Section V summarizes the findings and concludes the paper.

\section{An Overview of the Power Generation TECHNOLOGIES}

The future ship on-board MVDC electrical distribution systems, especially power generation applications, are expected to adopt different technologies from the state-of-the-art MVAC and LVDC electrical distribution systems. Moreover, certain equipment, e.g., gearboxes and bulky transformers for voltage coordination are expected to be removed in the MVDC electrical distribution systems to provide flexibility and footprint reduction in the overall ship design [18]. This evolution of the on-board electrical distribution system, from the state-of-the-art MVAC to prospective MVDC system, is illustrated in Fig. 1 with notional MVAC and MVDC systems. In the MVAC systems, fixed speed prime movers and generators are the norm, producing electrical power at constant ac frequency and supplying different loads through different switchboards and voltage coordination transformers [5]. These multi-pulse transformers are especially important for connecting the variable speed drives (VSDs) for propulsion, which are often the major loads of the installation. The MVDC distribution provide possibilities to reduce the equipment footprint, increase energy density and efficiency of the system, as it allows flexibility in design by offering choice of selection between various standard or non-standard power conversion equipment [19].

In the following subsections, a brief discussion is provided on

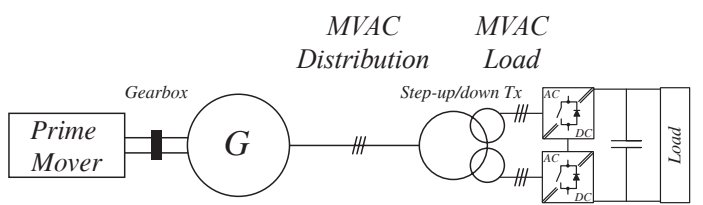

(a)

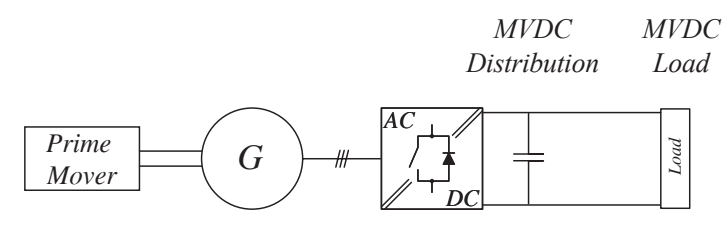

(b)

Fig. 1. (a) Simplified notional MVAC distribution with gearbox interfacing prime mover and generator, and multi-pulse transformer connected to the propulsion VSD. (b) Simplified notional MVDC distribution where gearbox and transformer are removed.

various equipment, commercially available (used), with considerations on their role in the power supplies of the future MVDC ships.

\section{A. Prime Movers}

The prime mover-generator set is the main source of power for the on-board electrical power systems. Internal combustion engines (ICEs) are usually used as prime movers in many marine applications. These run on diesel or heavy fuel oil (HFO), but wherever gas is available as a cheaper alternative gas turbines, steam turbines or combined cycle turbines can also be found, especially for high speed vessels or liquefied natural gas (LNG) tankers [5]. In an effort to improve the fuel efficiency and reduce emissions of ICEs, ABB has introduced dynamic ac (DAC) and LVDC electrical distribution for large and medium size ships showing $6 \%$ and $20 \%$ reduction in fuel consumption, respectively [2], [20]. Another opportunity, arising from the use of variable speed operation of the prime movers and lack of requirement for the synchronization of the generators, is the consideration of high speed ICEs and gas turbines as the principle prime movers for high speed generators. This presents the possibility to remove step up/reduction gearboxes, interfacing the prime movers and the generators, considering that their efficiency is around $70 \%$ and their size is considerable at high power levels [21]. A summary of the available prime mover technologies is provided in TABLE I.

1) Internal Combustion Engines: ICEs, used as prime movers in the state-of-the-art marine MVAC electrical distribution systems, are usually operated at medium (400$1000 \mathrm{rpm}$ ) to high speed speeds (1000 rpm and above) [28]. Mainly liquid fuel, e.g., HFO or marine diesel oil, is used, but some applications also utilize gas, e.g., LNG tankers. Additionally, ICEs having dual fuel capability are also being employed, in accordance with the Tier II and Tier III emission reduction programs of International Maritime Organization (IMO) [23]. There is a constant push to improve efficiency and emissions of the ICEs, however, the opti- 
TABLE I

Specifications of ICEs and Gas Turbines for Marine Applications

\begin{tabular}{|c|c|c|c|c|c|c|}
\hline PM & Manufacturer & Power Range & Thermal Efficiency & Speed & Fuel Type & Reference \\
\hline \multirow{4}{*}{ ICE } & Wärtsilä & $0.92-11.2 \mathrm{MW}$ & $42-52 \%$ & $600-1000 \mathrm{rpm}$ & Liquid/Gas & {$[22]$} \\
\hline & MAN & 0.45-12 MW & $48-55 \%$ & $720-1800 \mathrm{rpm}$ & Liquid/Gas & [23] \\
\hline & GE & 1.3-4.7 MW & $\approx 50 \%$ & $900-1050 \mathrm{rpm}$ & Liquid/Gas & {$[24]$} \\
\hline & Rolls Royce & 3.6-9.6 MW & $44-48.5 \%$ & 720/750 rpm & Liquid/Gas & {$[25]$} \\
\hline GT & GE & 4.5-42 MW & $\approx 40 \%$ & $7-3.6 \mathrm{krpm}$ & Gas & {$[26]$} \\
\hline $\begin{array}{l}\text { ICE } \\
\text { GT }\end{array}$ & Literature & $\begin{array}{l}\text { 1-10 MW } \\
\text { 3-40 MW }\end{array}$ & $\approx 40 \%$ & $\begin{array}{l}\text { 720/750 rpm } \\
3-15 \mathrm{krpm}\end{array}$ & Liquid/Gas & {$[5]$} \\
\hline
\end{tabular}

TABLE II

Specifications of Generators for Marine Applications

\begin{tabular}{|c|c|c|c|c|c|}
\hline Gen & Manufacturer & Power Range & Voltange & Speed & Reference \\
\hline \multirow{3}{*}{ SM } & $\mathrm{ABB}$ & 1-50 MVA & $1-15 \mathrm{kV}$ & $500-1800 \mathrm{rpm}$ & {$[30]$} \\
\hline & Siemens & $0.38-20 \mathrm{MW}$ & $0.4-13.8 \mathrm{kV}$ & $500-1800 \mathrm{rpm}$ & [31] \\
\hline & GE & $2.5-45 \mathrm{MW}$ & $0.4-15 \mathrm{kV}$ & $300-1200 \mathrm{rpm}$ & [32] \\
\hline
\end{tabular}

mum fuel consumption of a medium speed ICE is still very high for constant speed operation in MVAC distribution (around $200 \mathrm{~g} / \mathrm{kWh}$ for $85 \%$ loading). This can be reduced to around $70 \mathrm{~g} / \mathrm{kWh}$ for a variable speed operation $(50 \%$ $100 \%$ rated speed), which is possible using de distribution [2]. Decrease of fuel consumption and operational costs is one of the key commercial motivations for move from MVAC to MVDC ships.

2) Gas Turbines: GTs have been deployed in military ships since 1960s and are also finding their way into commercial vessels as auxiliary generators [5]. They are increasingly being considered, as the main prime movers in ships, for power generation as they are reliable and have a smaller footprint compared to ICEs, therefore, ensuring energy density. However, they have rather low efficiency $(\approx 40 \%)$ and are expensive to run [29]. In the future MVDC system, GTs are expected to be deployed to operate on wide speed range which improves their efficiency, system performance and their compactness also ensures energy density and space saving. Different manufacturers like GE and Rolls Royce are offering different gas turbines ranging from 3-42 MW for marine applications [26], [27].

\section{B. Generators}

Another important part of the on-board electric power generation are the electrical machines, operated as generators. In ac systems, these are coupled, either directly or through a gear box, to the shaft of the prime movers that rotate at a constant speed of $720 / 750 \mathrm{rpm}$ in case of medium speed diesel engines and upto $1800 \mathrm{rpm}$ for high speed diesel engines. Like the prime movers, dc electrical power distribution allows the generators to operate at variable ac frequencies, removing the need for synchronization of different generators on the ship. This allows the system designers to consider high speed generators, e.g., permanent magnet synchronous generators (PMSG), matching the speed of the prime mover and complete removal of the gearbox. A brief summary and key characteristics of a few selected generators are given in TABLE II.

1) Synchronous Generators: The most commonly used generator technology, in the on-board electrical distribution systems, is the synchronous generator [5]. These generators usually have $8 / 10$ poles and rotate at speed of $750 / 720 \mathrm{rpm}$ to produce ac voltage at $50 / 60 \mathrm{~Hz}$. Generators, with 2, 4 or 6 poles, are also sometimes employed and rotate at their respective speed to produce $50 / 60 \mathrm{~Hz}$ ac. As prime mover and generator rotation speeds are usually not the same, a gearbox is needed to have the correct rotational speed of the generator. Automatic voltage regulators (AVRs) control the excitation current of the field windings (either as direct or brushless excitation) in order to maintain the terminal voltage of the generators. For steady state operations, the voltage regulation is limited to $\pm 2.5 \%$ of the nominal voltage, while, voltage transients must not exceed $-15 \%$ or $+20 \%$ of the nominal generator voltage. Oscillations, in the generator voltage due to load sharing among different generators or any load variations, are normally damped by the damper windings [5].

2) Permanent Magnet Synchronous Generators: Permanent magnet synchronous machines (PMSMs) are mainly deployed in oil and gas industry as turbo compressors [33], but their use as possible generators for dc power systems has been reported in literature [34], [35]. PMSMs can be used in applications where high speed operation, i.e., 8000 rpm and above, is required [35]. The high frequency operation of PMSM is possible due to the use of permanent magnets for magnetic field rather than externally provided excitation for the rotor field winding. Despite the benefits of using the PMSMs, there are certain challenges due to 
the lack of long operational experience in marine applications, and the full potential of PMSMs is difficult to assess/ realize, as summarized in [7].

\section{Transformers}

Transformers, in the marine MVAC electrical distribution networks, are mainly used for the reason of galvanic isolation and voltage coordination, considering that rated voltage of electrical generators does not necessarily match the voltage of propulsion motors [5]. These transformers, usually found at the input of propulsion VSDs or large group of LVAC loads, are bulky, heavy and occupy significant space in the already constrained area. Due to use of oil for cooling and insulation and in case of faults, they are a fire hazard and other environmental concerns. However, these transformers usually have multiple phase shifted secondaries and are interfaced to multi-pulse rectifiers providing high quality ac supply to the propulsion inverters, while simultaneously improving the power quality indices (current and voltage distortions) and reducing the negative impact on other components of the marine electrical power system, e.g., generators. At the same time, they provide short circuit limiting impedance in case of faults. IEC standards are also available for design of marine transformers depending on their functionality [36]. While, there is a clear motivation to remove transformers from the future marine MVDC electrical distribution networks, it would be advantageous to preserve possibility to use multipulse rectifiers for quality of MVDC supply. For that reason, multi-phase generators are seen as important technology that can provide the same function, even though it is not the exact replacement technology.

\section{Rectifiers}

Even though rectifiers, as standalone equipment, are not essential part of MVAC ship design, discussion is provided hereafter, as their importance will be significant for MVDC ships. Presently, state-of-the-art VSDs, employed in the on-board MVAC distribution systems in ships, have their own rectifiers as integral part of the package, and since regeneration is usually not needed these are often simple multi-pulse diode rectifiers. In the new ship on-board MVDC electrical distribution system, rectifiers become essential part as they are directly interfaced to generators, provide conversion from ac to dc, and directly impact the quality of the MVDC supply. As this removes the need for synchronization of different generators, it also provides a lot of flexibility in system design and opportunity to use energy dense solutions, such as high speed generators. Various rectifiers topologies, such as diode, thyristor, active (classical 2-level/ multi-level topologies) or MMC rectifiers can be considered and their benefits should be analyzed on the system level.

1) Diode Rectifier Unit: The schematics of the diode rectifier unit (DRU), with a capacitive filter, is shown in Fig. 2(a). As it is made of passive devices, it lacks the ability to regulate the dc-side voltage or the ac-side currents, but this makes its construction the simplest and cheapest among the different rectifier topologies. The DRUs are employed

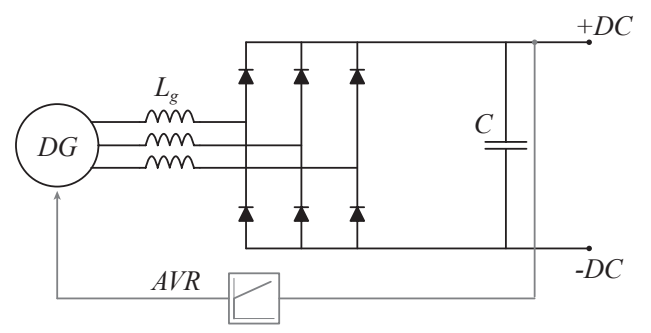

(a)

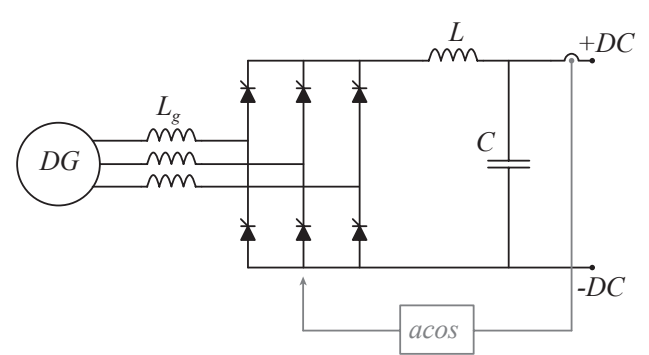

(b)

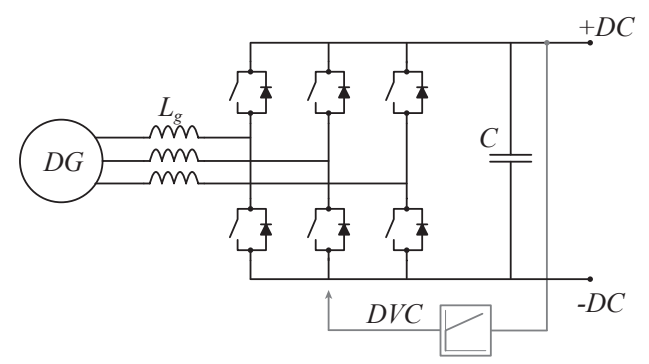

(c)

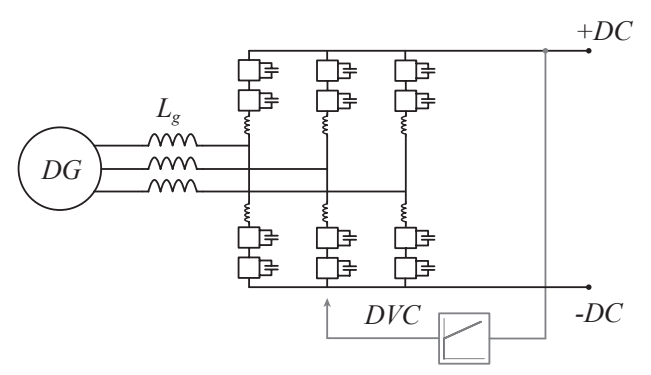

(d)

Fig. 2. 3-phase system configuration with different source side converters. (a) Diode rectifier. (b) Thyristor rectifier. (c) Multi-level active rectifier. (d) MMC active rectifier.

in the present drive technology [37], especially for marine applications where no regeneration is possible, and are interfaced with input multi-secondary transformers in 12, 18 or 24-pulse series or parallel configurations, but also in the new LVDC marine systems [4].

2) Thyristor Rectifier Unit: The second type of rectifier that can be considered for MVDC supply is the thyristor rectifier unit (TRU), as shown in Fig. 2(b). So far, in marine on-board distribution (except LVDC) they have not been used, but as they provide possibility to regulate the dc-side voltage in a narrow voltage range, they are being considered as a possible MVDC supply side converter [16], [38]. Furthermore, they are being deployed for LVDC 
electrical distribution systems as supply side converters [2], even though during normal conditions they are operated as DRUs. TRUs have a more complicated construction compared to DRUs and are more expensive alternative to DRUs. However, in the LVDC distribution systems, TRUs provide possibilities to reduce fault current due to ability to reverse output dc voltage [2] and for those reasons TRUs are also considered for the MVDC electrical distribution systems [16].

3) Active Rectifier Unit: The active rectifier unit (ARU) with a capacitive filter is shown in Fig. 2(c) and it represents the most advance solution. Different ARU topologies, e.g., classic 2-level voltage source converter (VSC) and multi-level like 3-L neutral point clamped (NPC), 3-L active NPC (ANPC), 4-L flying capacitor (FC) and 9-L cascaded h-bridge (CHB) are possible and also found in the commercially available products. Among these topologies, the 3-L NPC is most widely used in MV applications [39]. ARUs are relatively expensive compared to the DRUs and TRUs and their ability to allow bidirectional power flow is of limited use, as the regeneration is normally not possible. Additionally, ARUs cannot control currents in case of faults [40], hence, their application in marine systems does not bring much advantage (except for possibility to tightly regulate dc bus voltage), considering system perspective.

4) Modular Multilevel Converter: The modular multilevel converter (MMC) (Fig. 2(d)), can be classified as another ARU technology that can be considered for marine MVDC applications as it offers certain advantages: higher efficiency due to lower switching frequency, modularity, voltage scalability and reduced filtering effort due to the presence of sub-modules [41]. The bipolar sub-modules also help in limiting the dc-side fault currents by the converter itself. MMC is reported as a possible supply side converter for marine MVDC electrical distribution systems in [42]. However, MMCs are likely more complicated solution than needed in reality, and especially since their footprint is normally bigger than commercial ARU based on multi-level topologies. Yet, they do offer better scalability and flexibility to adapt to different voltage requirements in MVDC systems.

While different rectifier topologies could be analysed, DRUs are considered in this work for a number of reasons: (i) they are already employed in the commercial marine VSDs with significant operational experience and design knowledge, (ii) TRUs do not have substantial advantages over DRUs unless fault limiting features are highly valued and desired, (iii) ARUs and MMCs, despite being the most advanced four-quadrant solution, are likely too costly for real commercial considerations. Additionally, even without sophisticated control, the dynamic performance of DRUs is also very good for marine applications [38], [43].

\section{Multi-Phase Multi-Pulse Marine MVDC Supplies}

The expected benefits of the MVDC electrical distribution system, e.g., fuel efficiency, lower operation costs, energy den- sity and smaller footprint to name a few, revolve around the technologies and operation strategies chosen for the MVDC supplies. Marine MVDC supplies are expected to power systems with different distribution voltage levels such as 5,10 , $15,20,25,30$ and $35 \mathrm{kV}$, depending on their power levels [1]. There are already concepts which allow for generator frequency variation in MVAC systems (e.g., 48-60 Hz) such as dynamic AC (DAC) [20], with goal to maximize the efficiency. Howev$\mathrm{er}$, in terms of prime-mover rotational speed or generator ac frequency, much larger variations are expected in MVDC systems (e.g., 50-1000 Hz), when compared to present MVAC systems.

\section{A. 3-Phase Electrical Generation}

On the system level and based on previous discussions different configurations are possible for the prime movers, generator types, ac and dc voltage levels, operating frequencies, and type and control of the supply-side converter. However, increase of the power levels is normally followed by the increased MVDC voltage, and considering desire to avoid use of transformers in the system, typical 3-phase generators connected to simple 6-pulse rectifiers have certain shortcomings. Limited blocking voltages of present semiconductors require more devices to be connected in series and this increases the complexity of the auxiliary circuits like voltage balancing circuits and snubbers. Power quality of the 6-pulse rectifiers is also quite low [44], and there is no simple way to implement redundancy, other than redundancy on the system level as it is the case already. Despite being simple and reliable solution, it does not provide sufficient voltage scalability, and for those reasons other solutions are required.

\section{B. Multi-Phase Electrical Generation}

To overcome previously described challenges, multi-phase generators characterized with multiple sets of 3-phase windings should be considered. These machines are mostly employed as propulsion motors due the redundancy offered by the multiple sets of 3-phase windings. This redundancy, while of no use in MVAC distribution due to requirement of generator synchronization, has a considerable potential in the supplies for the future MVDC systems. Multi-phase generators coupled with multiple 6-pulse DRUs appear as an attractive solution. Moreover, these multi-pulse DRUs, e.g., 12, 18, 24-pulse depending on the voltage or power requirements of the system, could be built from multiple basic 6-pulse DRU units [37]. The advantages expected from these multi-pulse multi-phase supplies are: high quality of MVDC voltage achieved in a simple way; multi-phase generator to some extent replaces the role of multi-winding transformer; better fault tolerance and introduction of another level of redundancy in the system; provide additional degree of freedom to select voltage classes of generators and motors; ability to utilize present semiconductor technology in an efficient way. Two possible arrangements of these configurations are shown in Fig. 3 and discussed below.

1) Multi-Pulse Parallel Rectifiers: The first configuration under consideration is the parallel connection of the rectifiers, as shown in Fig. 3(a). The voltage rating of the 


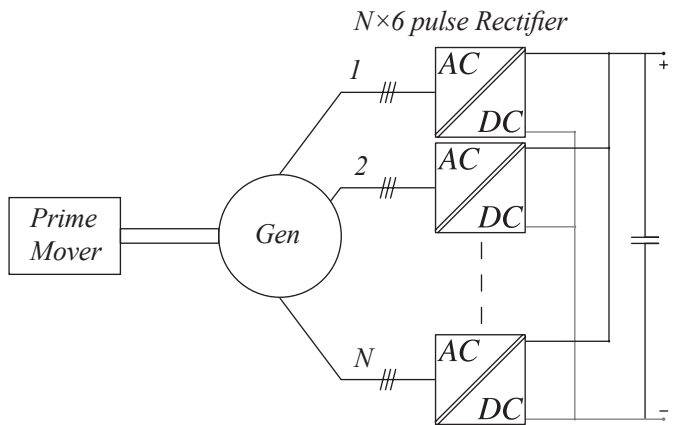

(a)

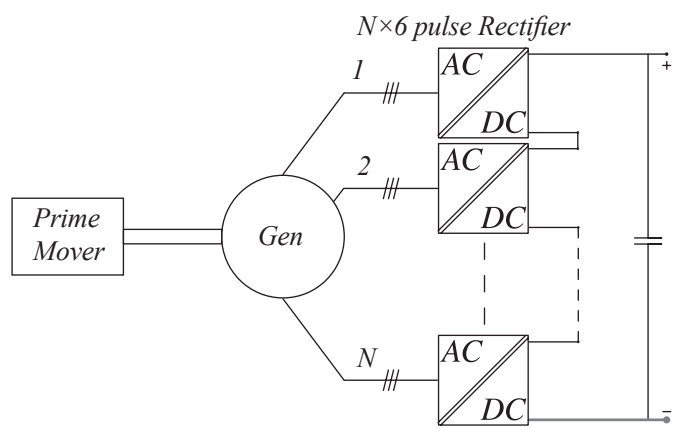

(b)

Fig. 3. N×3-phase system configuration. (a) Parallel connection of rectifiers. (b) Series connection of rectifiers.

windings of the $\mathrm{N} \times 3$-phase generator are equal to the full ac-side voltage, e.g., $3.7 \mathrm{kV}$ ac for $5 \mathrm{kV}$ dc-side supply or $7.4 \mathrm{kV}$ for $10 \mathrm{kV}$ dc-side supply. However, the total current is divided among the different winding sets i.e., $I_{a c, N}=$ $I_{\text {rated }} / N$, where $\mathrm{N}$ is the number of 3-phase winding sets of the generator. Moreover, each rectifier is also rated to the full dc-side voltage, and depending on the exact MVDC voltage level $(10 \mathrm{kV}, 15 \mathrm{kV}, \ldots$ and selected voltage class of semiconductor devices $(4.5 \mathrm{kV}, 6.5 \mathrm{kV})$ there may be a need for a series connection of several devices (including snubbers and static and dynamic voltage balancing circuits). Similar to the case of the generator winding sets, the current through each rectifier is $I_{r e c, N}=I_{d c, t o t} / N$. The parallel connections of the rectifiers, albeit providing a high quality MVDC supply, eventually leads to situation where the voltage class of the generators and motors are similar/ identical. The assumption here is that motors (propulsion or other pumps) are connected to MVDC electrical distribution network through a dedicated inverter. However, in case of a fault resulting in loss of a 3-phase winding set or one of the rectifiers, the system can continue to operate in a de-rated mode. This means all critical loads can be supplied, while, non-essential loads are disconnected. Thus multi-phase multi-pulse parallel rectifiers are suited for ship architecture where fault tolerance is of high importance.

2) Multi-Pulse Series Rectifiers: The series connection of several rectifiers is shown in Fig. 3(b). In this case, the voltage rating of each of the generator winding set and rectifier is $V_{a c / d c, N}=V_{a c / d c, t o t} / N$. However, as the load current
TABLE III

N×3-Phase System Level Design - Non Isolated - No Gearbox - N×3Phase Generator - Target 5 -35 kV MVDC

\begin{tabular}{|c|c|c|c|c|}
\hline \multirow[t]{2}{*}{ PM } & \multicolumn{2}{|c|}{ Generator } & \multicolumn{2}{|l|}{ Rectifier } \\
\hline & Phase No. & Vltage (V) & Config & MVDC \\
\hline \multirow{7}{*}{ Any } & 6 & $\begin{array}{c}2 \times 3 \times 3.7 \mathrm{kV} \\
2 \times 3 \times 1.85 \mathrm{kV}\end{array}$ & $\begin{array}{l}\text { DRU,12-p.P } \\
\text { DRU,12-p.S }\end{array}$ & \multirow{7}{*}{$5 \mathrm{kV}$} \\
\hline & 0 & $3 \times 3 \times 3.7 \mathrm{kV}$ & DRU,18-p.P & \\
\hline & 9 & $3 \times 3 \times 1.23 \mathrm{kV}$ & DRU,18-p.S & \\
\hline & \multirow{2}{*}{12} & $4 \times 3 \times 3.7 \mathrm{kV}$ & DRU,24-p.P & \\
\hline & & $4 \times 3 \times 925 \mathrm{~V}$ & DRU,24-p.S & \\
\hline & \multirow{2}{*}{15} & $5 \times 3 \times 3.7 \mathrm{kV}$ & DRU,36-p.P & \\
\hline & & $5 \times 3 \times 740 \mathrm{~V}$ & DRU,36-p.S & \\
\hline \multirow{6}{*}{\multicolumn{2}{|c|}{$\begin{array}{l}\text { Higher phase numbers and } \\
\text { voltages affects rectifier } \\
\text { arrangement }\end{array}$}} & \multirow{6}{*}{ Similar to above } & \multirow{6}{*}{$\begin{array}{c}\text { Parallel or } \\
\text { Series }\end{array}$} & $10 \mathrm{kV}$ \\
\hline & & & & $15 \mathrm{kV}$ \\
\hline & & & & $20 \mathrm{kV}$ \\
\hline & & & & $25 \mathrm{kV}$ \\
\hline & & & & $30 \mathrm{kV}$ \\
\hline & & & & $35 \mathrm{kV}$ \\
\hline
\end{tabular}

TABLE IV

Rectifier Module Parameters Considered in this Study

\begin{tabular}{cc}
\hline \hline \multicolumn{2}{c}{ Module Parameters } \\
\hline Rated Power $\left(P_{n}\right)$ & $9 \mathrm{MW}$ \\
Rated DC Voltage $\left(V_{d c}\right)$ & $5 \mathrm{kV}$ \\
Rated AC Voltage $\left(V_{a c}\right)$ & $3.7 \mathrm{kV}$ \\
Rated Frequency $\left(f_{n}\right)$ & $50 \mathrm{~Hz}$ \\
Generator Inductance $\left(L_{g}\right)$ & $\frac{V_{d c}^{2}}{2 \pi f_{n} S_{n}} \mathrm{mH}$ \\
\hline Cable Parameters & \\
\hline Cable Inductance $\left(L_{c}\right)$ & $0.347 \mathrm{mH} / \mathrm{km}$ \\
Cable Resistance $\left(R_{c}\right)$ & $0.089 \Omega / \mathrm{km}$ \\
Cable Capacitance $\left(C_{c}\right)$ & $0.307 \mu \mathrm{F} / \mathrm{km}$ \\
\hline \hline
\end{tabular}

flows through each of the 3-phase winding set and rectifier, the current ratings is $I_{a c, N}=I_{\text {rated }}$. The series connection of the rectifiers lowers the required voltage class of the semiconductors, compared to the parallel case, providing more degrees of freedom for the optimization. As devices are expected to block lower voltages, this leads to fewer auxiliary circuits, e.g., snubbers and voltage balancing circuits. Another advantage which this configuration brings is the additional flexibility to optimize the selection of the voltage classes of generators and motors, i.e., generators with lower voltage ratings can supply propulsion motors with high voltage ratings (assuming appropriate insulation coordination on the generator side). However, parallel configuration does not provide redundancy, and in case of fault on any of sub-rectifiers, likely complete supply chain will be out of service. Bypassing faulty sub-rectifier would allow system to continue to operate, but since the MVDC voltage is reduced, this is of little practical relevance.

TABLE III provides a summary of the possible voltage 
TABLE V

Design of 9 MW 6-Pulse Rectifier Module

\begin{tabular}{|c|c|c|c|}
\hline \multicolumn{2}{|c|}{ Device } & \multirow{2}{*}{$\frac{\text { Parameters }}{V_{R R M}(V)}$} & \multirow{2}{*}{$\frac{\text { 6-Pulse Rectifier Module }}{1310 @ 2 \text { devices connected in series }}$} \\
\hline Manufacturer & $\mathrm{ABB}$ & & \\
\hline Model & 5SDD06D600 & $I_{\text {davg }}(A)$ & 300 \\
\hline$V_{R R M}(V)$ & 6000 & $I_{\text {dpeak }}(A)$ & 910 \\
\hline$I_{\text {favg }}(A)$ & 662 & $I_{d r m s}(A)$ & 520 \\
\hline$I_{\text {frms }}(A)$ & 1040 & No. of devices & 24 \\
\hline$I_{F S M}(A)$ & 10500 & Loss per device $(W)$ & 740 \\
\hline$v_{f}(V)$ & 1.066 & Total Loss $(W)$ & 17700 \\
\hline$r_{t}(m \Omega)$ & 0.778 & Efficiency & $99.5 \%$ \\
\hline
\end{tabular}

classes of the multi-phase generators to achieve a certain dc-side voltage. A variety of combinations are possible, providing flexibility for the ship designers to optimize the overall system and carry out voltage coordination between generators and motors.

\section{Design, Simulation and Comparison of the MVDC SUPPLIES}

In this section, design of a 6-pulse DRU sub-module is presented, which then can be arranged in parallel and series configurations to achieve high quality MVDC supplies for different power levels and distribution voltages to demonstrate the effectiveness of multi-phase multi-pulse rectifiers. Furthermore, fault withstand capabilities of these supplies are investigated and need for fast protection is elaborated, considering the lack of DC breaker technology, fast power electronics fuses could be considered for protection of the rectifiers in case of faults.

\section{A. Design of DRU Sub-Module}

In order to realize different MVDC supplies, a $5 \mathrm{kV}$ 6-pulse DRU module is designed for parameters given in TABLE IV, using $\mathrm{ABB}$ diodes rated at $6 \mathrm{kV}$ blocking voltage [45]. The blocking voltage requirement for the devices is calculated using $V_{R S M}=\sqrt{2} V_{S, r m s} \times k$, here $V_{R S M}$ is absolute maximum rating of the diode, $V_{S, r m s}$ is the rms value of the ac voltage and $k$ is safety factor usually taken as 2.5 [46]. For this case, devices are required to block $13.1 \mathrm{kV}$ which translates into connection of 2 devices (with auxiliaries) in series to fulfill this requirement. Additionally 2 more devices, in similar arrangement, are required to be connected in parallel for the rectifier to withstand high fault currents (discussed later w.r.t. $I^{2} t$ ), leading to $4(2+2)$ devices per branch of the DRU sub-module. Schematics of the DRU sub-module are given in Fig. 4. Furthermore, these parallel-connected devices are considered to have minimal discrepancies in their $v_{f}$ and $r_{t}$, respectively. This ensures that the current is almost evenly shared by the 2 sets of diodes. The device parameters and sub-module losses and efficiency, for the operation, at rated power are given in TABLE V.

As losses are incurred at each device, the device junction temperature $\left(T_{j}\right)$ should not exceed the maximum junction temperature limit $T_{j \max }$ specified in the data sheet and, in this

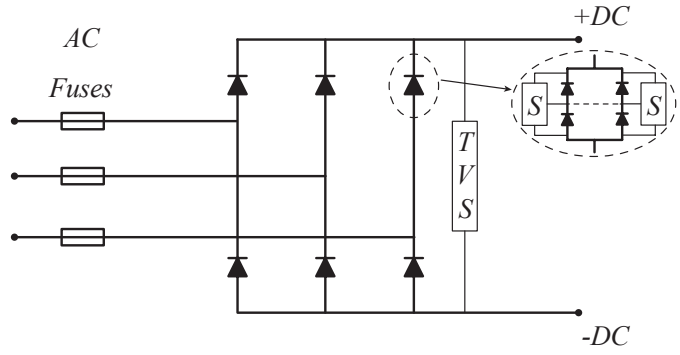

Fig. 4. DRU sub-module with snubbers (S) and transient voltage suppression (TVS) circuts.

case, $T_{j \max }$ is $150^{\circ} \mathrm{C}$ [45]. The temperature difference between junction and heat sink can be calculated using $\Delta_{J H}=P_{\text {loss }} \times\left(R_{t h j c}\right.$ $\left.+R_{t h, c h}\right)$, here $R_{t h, j c}$ and $R_{t h, c h}$ are the thermal resistance from the junction to the case and the case to the heat sink, respectively. For the losses given in TABLE V (calculated using procedure specified in [47]), and $R_{t h, j c}=42 \mathrm{~K} / \mathrm{kW}$ and $R_{t h, c h}=8 \mathrm{~K} / \mathrm{kW}$ from data sheet [45], the resultant $\Delta_{J H}=37{ }^{\circ} \mathrm{C}$. Considering $80{ }^{\circ} \mathrm{C}$ at the heat sink, $T_{j}=117{ }^{\circ} \mathrm{C}$ which is smaller than the $T_{j \max }=150{ }^{\circ} \mathrm{C}$ limit specified for each device. Therefore, the rectifier module has sufficient thermal ratings to operate at full load.

\section{B. Case 1: $5 \mathrm{kV}, 18 \mathrm{MW}$, Parallel 12-Pulse Rectifier}

The first case considers a medium speed diesel engine (720/750 rpm), operated in a DAC configuration for efficient fuel usage, driving a 6-phase generator ( $2 \times 3$-phase) interfaced to a parallel 12-pulse rectifier, as shown in Fig. 5(a). Each 3-phase winding set of the generator is rated at $3.7 \mathrm{kV}$ and 9 MW. The voltage rating of the generator also defines its insulation requirements. The connection of two $9 \mathrm{MW}$ rectifier modules in parallel generates a high quality $5 \mathrm{kV}$ dc-side voltage and also provides redundancy and fault tolerance in the system. In case of any generator or rectifier fault, e.g., failure of one of the 3-phase winding set or rectifier module, the system can be operated in a de-rated mode, i.e., non-essential loads can be disconnected from the system. The LC-filter at the output of the rectifier is designed according to the steps highlighted in [47]. The ac-side input voltage and current, and dc-side voltage and current of the each DRU sub-module are given in Fig. 6(a), Fig. 6(c) and Fig. 6(e), whereas, dc-side voltage and current for the complete rectifier system supplying a $18 \mathrm{MW}$ load are 


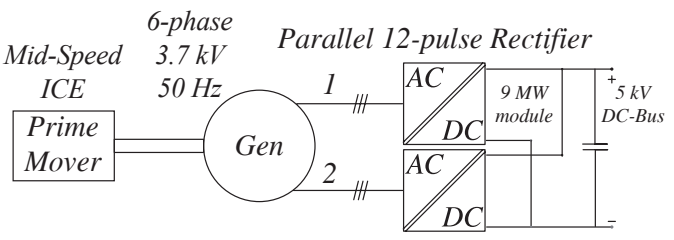

(a)

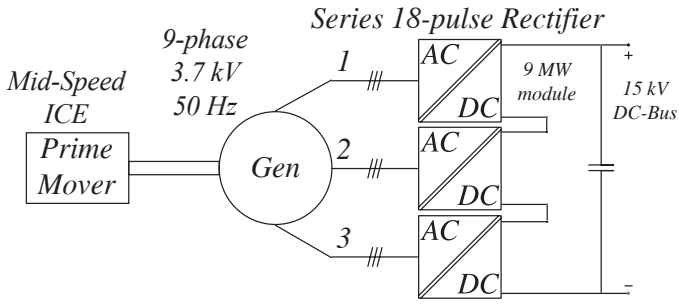

(b)

Fig. 5. Proposed multi-phase multi-pulse configuration. (a) Case 1: $5 \mathrm{kV}, 18 \mathrm{MW}$, parallel 12-pulse rectifier based dc supply. (b) Case 2: $15 \mathrm{kV}$, $27 \mathrm{MW}$, series 18-pulse rectifier based dc supply.

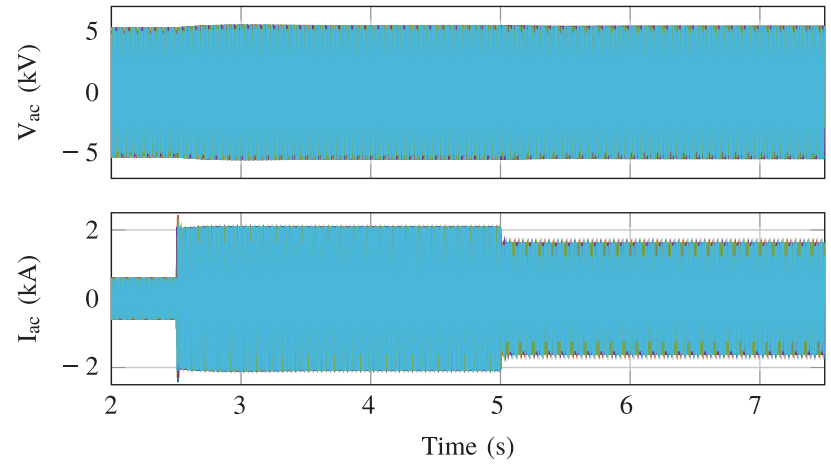

(a)
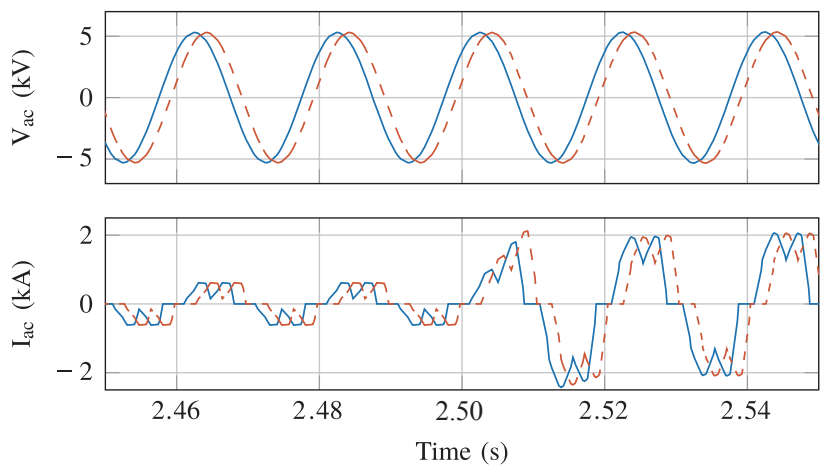

(c)

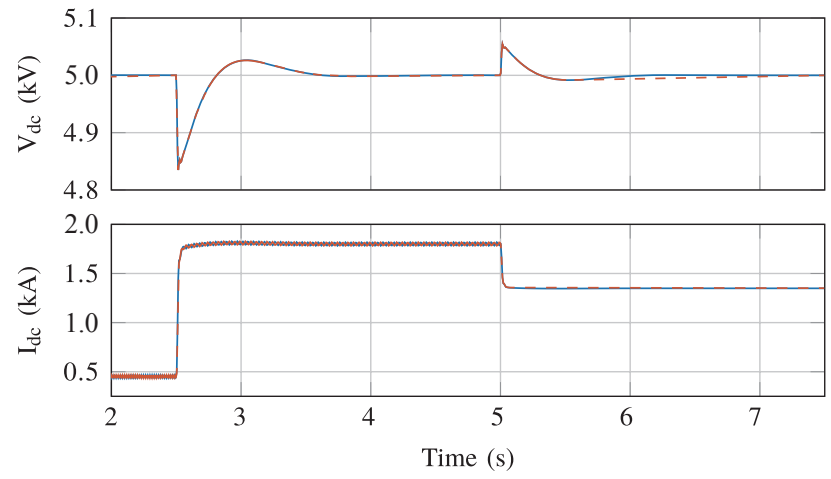

(e)
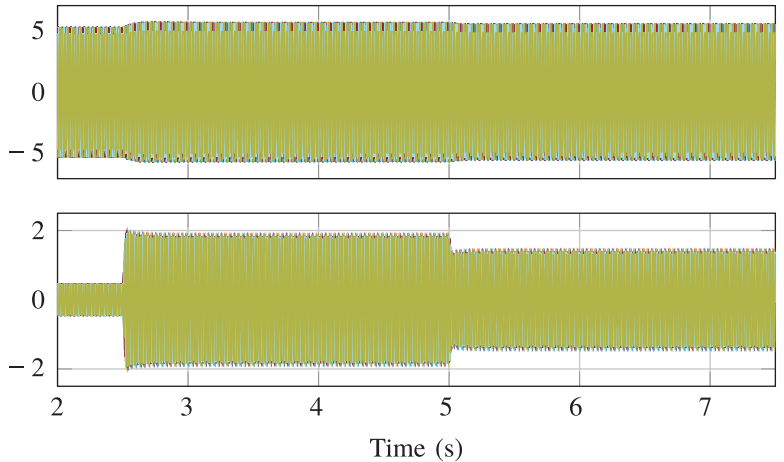

(b)
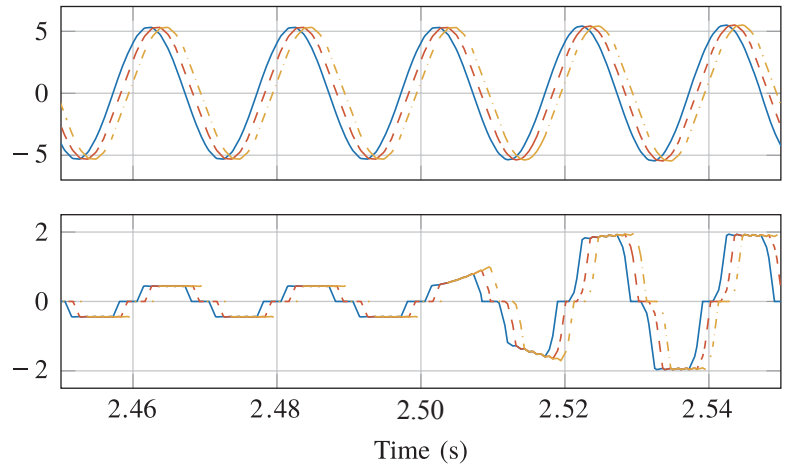

(d)

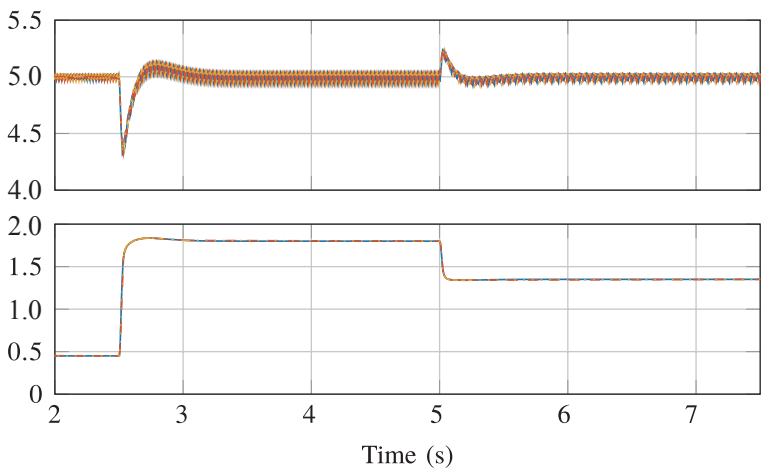

(f)

Fig. 6. The ac and dc-side voltage and current wave forms for DRU sub-modules for case 1 and case 2. (a) AC voltage and current for case 1 , highlighting envelope of the voltages and currents. (b) AC voltage and current for case 2, highlighting envelope of the voltages and currents. (c) Zoom in of the load change from $25 \%$ to $100 \%$ at $2.5 \mathrm{~s}$ (solid for phase $\mathrm{A}$ of the $1^{\text {st }}$ winding set and dashed for phase $\mathrm{A}$ of the $2^{\text {nd }}$ winding set). (b) Zoom in of the load change from $25 \%$ to $100 \%$ at $2.5 \mathrm{~s}$ (solid for phase $\mathrm{A}$ of the $1^{\text {st }}$ winding set, dashed for phase $\mathrm{A}$ of the $2^{\text {nd }}$ winding set) and dash-dotted for phase $\mathrm{A}$ of the $3^{\text {rd }}$ winding set). (e) DC voltages and currents for case 1 with solid for $1^{\text {st }}$ sub-module and dashed for $2^{\text {nd }}$ sub-module. (f) DC voltages and currents for case 2 with solid for $1^{\text {st }}$ sub-module, dashed for $2^{\text {nd }}$ sub-module and dash-dotted for $3^{\text {rd }}$ sub-module. 


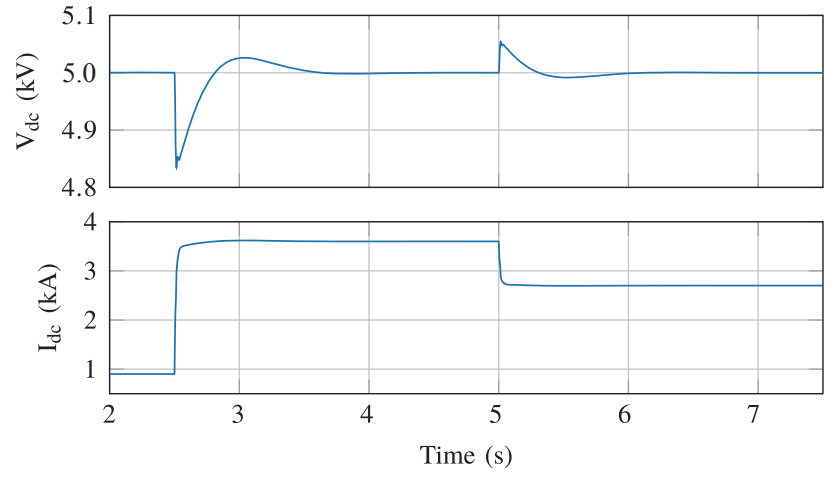

(a)

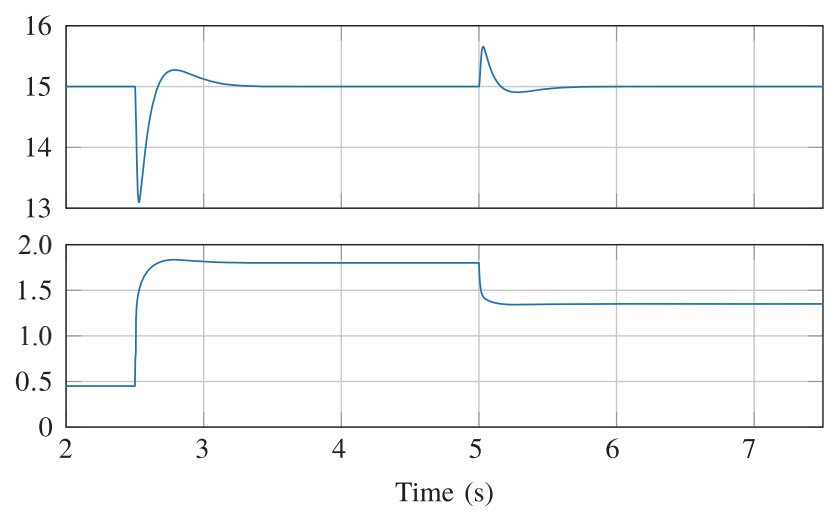

(b)

Fig. 7. Time domain simulations for (a) Case 1. (b) Case 2.

shown in Fig. 7(a). The load is changed from $25 \%$ to $100 \%$ at $2.5 \mathrm{~s}$ and then dropped to $75 \%$ at $5 \mathrm{~s}$. The only control working is the AVR of the generator which corrects the voltage.

\section{Case 2: 15 kV, 27 MW, Series 18-Pulse Rectifier}

The second system configuration also considers a medium speed diesel engine driving a 9-phase generator ( $3 \times 3$-phase) interfaced to a series 18-pulse rectifier (Fig. 5(b)). Each generator 3-phase winding set is rated for a voltage of $3.7 \mathrm{kV}$ and 9 MW. The diode rectifier module designed earlier is used here as well and 3 of it are connected in series to realize a $15 \mathrm{kV}$ dc supply rated at $27 \mathrm{MW}$. As discussed earlier, the series multi-pulse rectifiers provide flexibility when it comes to selecting voltage classes of the generators and the loads, e.g., here a generator rated at $3.7 \mathrm{kV}$ can supply a propulsion motor rated at $11 \mathrm{kV}$ without the requirement of a step up transformer. In case of any faults, e.g., generator or rectifier side faults, this configuration requires the system to be shutdown and repaired before supplying the loads again. The ac-side input voltage and current, and dc-side voltage and current of the each DRU sub-module are given in Fig. 6(b), Fig. 6(d) and Fig. 6(f), whereas, dc-side voltage and current for the complete rectifier system supplying a $27 \mathrm{MW}$ load are shown in Fig. 7(b). The load is changed from $25 \%$ to $100 \%$ at $2.5 \mathrm{~s}$ and then dropped to $75 \%$ at $5 \mathrm{~s}$. Again, only the AVR of the generator corrects the terminal voltage.

\section{Fault Withstand Capabilities}

Faults are inevitable to happen in complex systems and the different equipment present is characterized with fault withstand capabilities. Generally, power electronics equipment has much lower over-current withstand capability compared to other equipment. Thus, knowing surge current and thermal capabilities of all the equipment is of high importance for developing protection coordination strategies. In the state-of-the-art MVAC electrical distribution systems the initial short circuit current is usually 10-15 times the rated current. This current then settles to 3-5 times the rated current, in $500 \mathrm{~ms}-1 \mathrm{~s}$, when the ac circuit breaker is operated [5]. However, the surge current and thermal capabilities of power electronic devices are much lower than the generators, transformers, cables, and so on. This means that these devices are the bottlenecks for the protection coordination strategies. Following the physical settings of the LVDC system, as discussed in the introduction, the proposed MVDC supplies are considered to be equipped with multi-phase generators having an excitation removal system to lower the short circuit currents, even though this is very slow action, considering fault dynamics. Additionally, two scenarios are defined to test the proposed rectifier topologies for their fault handling capabilities considering a dc pole to pole fault. These different scenarios consider the removal of the generator excitation at $2 \mathrm{~ms}$, with a excitation time constant of $5 \mathrm{~s}$ :

- Generator with rated $\mathrm{X}_{g}$.

- Generator with 1.5 rated $\mathrm{X}_{g} "$.

The two scenarios test the impact of the excitation removal, higher $\mathrm{X}_{g}$ " and different distribution lengths on the fault energy and its implications on the fault withstand capability of the rectifiers. The 12-pulse parallel and 18-pulse series DRUs for 5 $\mathrm{KV}$ and $15 \mathrm{kV}$ MVDC distribution, respectively, are simulated for the above scenarios with a $10 \%$ fault impedance and fault at two different points in the distribution system. The simulations results of the dc-side voltage, currents and fault energy $\left(I^{2} t\right)$ are shown in Fig. 8. It can be observed from Fig. 8 that after the fault condition occurs at $2 \mathrm{~s}$, the fault currents are lower for a higher $\mathrm{X}_{g}$ " but this has negligible impact on the peak of the fault current, which is almost 10 times the rated current for both cases. Additionally the farther the fault is from the source, the higher is the impact of the cable, which results in a lower peak of the fault current. It is worth noting that the surge current after fault for 12-pulse rectifier reaches upto $31 \mathrm{kA}$, shown in Fig. 8(a), but it is shared among the two rectifier modules, whereas, for the 18-pulse rectifier where the initial surge is $17.6 \mathrm{kA}$, shown in Fig. 8(b), all the current flows through each of the modules. The surge current $I_{F S M}$, of the device selected to design these rectifiers, is given in TABLE $\mathrm{V}$ and has a value of $10.5 \mathrm{kA}$ for a 10 ms half-sine pulse. As two parallel connected devices are used in each module, their combined surge capability is $21 \mathrm{kA}$ for a $10 \mathrm{~ms}$ half-sine pulse, and in both cases the initial fault current pulses of $10 \mathrm{kA}$ and $4.5 \mathrm{kA}$ (10ms half-sine pulse) are below the surge current capability of the rectifiers. Thus, both rectifiers are sufficiently sized to handle initial fault currents. 

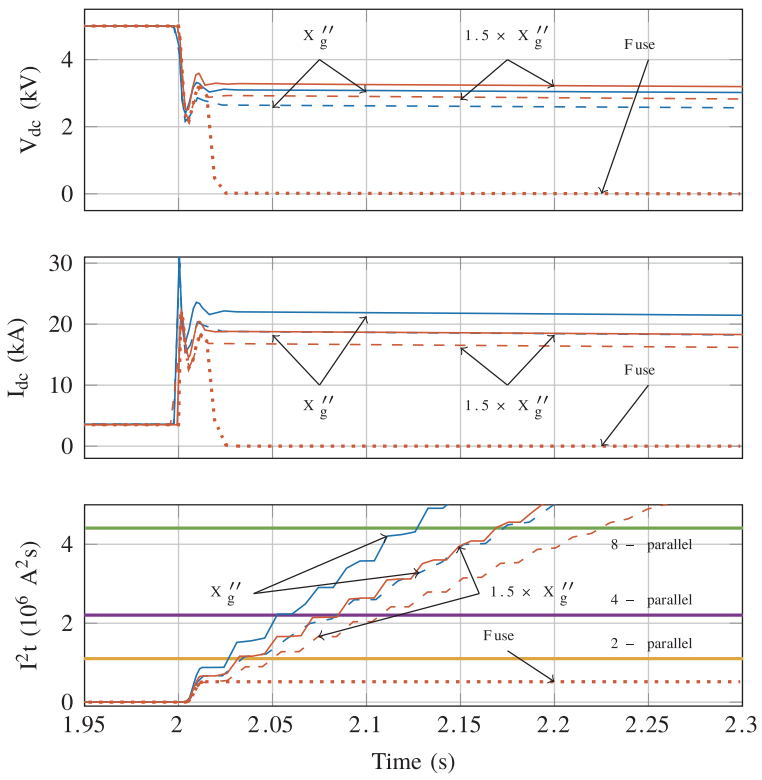

(a)
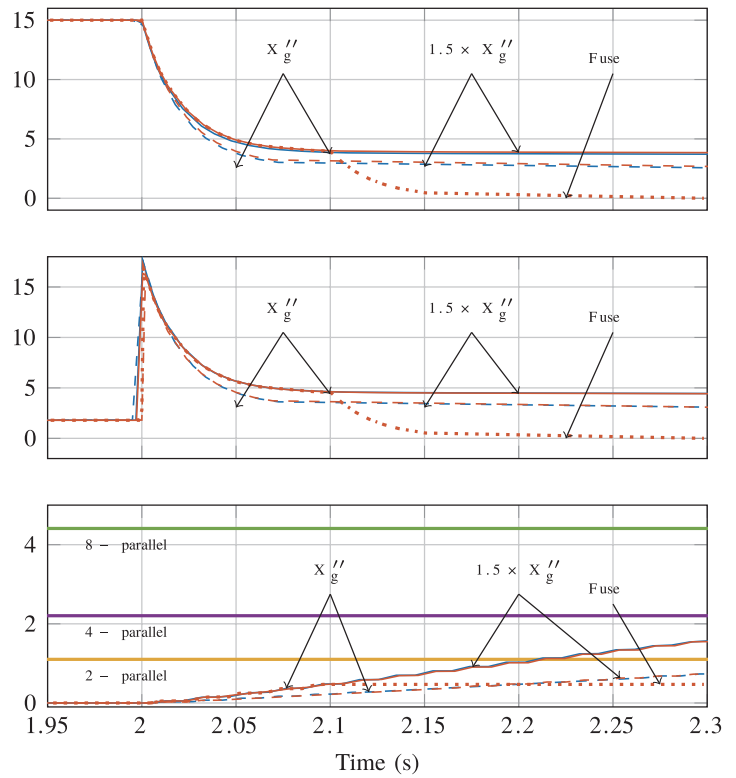

(b)

Fig. 8. Simulation results for dc-side pole to pole fault at $2 \mathrm{~s}$. Considering (i) Rated $\mathrm{X}_{\mathrm{g}}$ " with two different points for fault: at $10 \mathrm{~m}$ (solid line) and at $200 \mathrm{~m}$ (dashed line) from source, (ii) 1.5 Rated $\mathrm{X}_{\mathrm{g}}$ " with two different places for fault: at $10 \mathrm{~m}$ (solid line) and at $200 \mathrm{~m}$ (dashed line) from source and (iii) 1.5 Rated $\mathrm{X}_{\mathrm{g}}$ " with ac-side fuses (dotted line). A comparison of fault $\mathrm{I}^{2} \mathrm{t}$ with allowed $\mathrm{I}^{2} \mathrm{t}$ of two, four and eight parallel devices conductiong. (a) Case 1. (b) Case 2.

The bottom plots in Fig. 8 show the fault energy for the two scenarios, discussed earlier, and compares it with the thermal capabilities of different number of devices in parallel. It can be observed for Case 1, from Fig. 8(a), that the fault energy rises very fast, i.e., in $25 \mathrm{~ms}$ surpasses the combined $I^{2} t$ capacity of two parallel connected diodes. Even with higher $\mathrm{X}_{g}$ " and $L_{c}=$ $0.14 \mathrm{mH}$, the $I^{2} t$ capacity of two parallel connected diodes is surpassed in $50 \mathrm{~ms}$. It can also be observed that even connection of 8 diodes in parallel is not enough to with stand the fault energy and, therefore, other means are required to protect this rectifier. For Case 2, Fig. 8(b), the rise of fault energy is rather slow, compared to Case 1 , and but it still surpasses the $I^{2} t$ of the diodes in around $200 \mathrm{~ms}$. Similar to Case 1, external means are required to protect this rectifier as the fault current only goes to zero after several seconds and connecting several devices in parallel might not sufficient to with the fault energy in this time. For the analysis, number of diodes in parallel have been considered, while in reality the problem could be analyzed from the view of DRUs in parallel.

One inexpensive way to protect these rectifiers would be to consider fuses on the ac-side (no dc-side fuses because there is no regeneration) that can melt in a few milliseconds. These fast fuses also limit the fault current and the resultant energy through the diodes. In order to protect the rectifiers, in Case 1 and Case 2, these fuses must act fast enough to protect the rectifiers for both high and low fault currents. For Case 1, the fault current is 21.5 $\mathrm{kA}$ for for rated $\mathrm{X}_{g}{ }^{\prime}$ and $L_{c}=7 \mu \mathrm{H}$, however, with higher $\mathrm{X}_{g}{ }^{\prime \prime}$ and $L_{c}=0.14 \mathrm{mH}$, the fault current is lower, i.e., $16.6 \mathrm{kA}$. As in both scenarios the fault current are relatively high, the fuses selected must be fast enough and sized adequately to act within a few milliseconds. For Case 2, the fault current is $5 \mathrm{kA}$ for rated $\mathrm{X}_{g}{ }^{\prime \prime}$, while, a higher $\mathrm{X}_{g}{ }^{\text {" }}$ reduces it to $3 \mathrm{kA}$, irrespective of cable impedance. In both scenarios, the fault current is close to the rated current of $1.8 \mathrm{kA}$, i.e., 2.7 and 1.7 times, respectively.

In both cases, to protect the rectifier the ac-side fast fuses must be able to withstand rated current and voltage, under normal conditions, and act fast enough to block the fault currents, i.e., their $I^{2} t$ must be lower than the of the diodes $I^{2} t$ for the same currents. The impact of fuses on currents and voltages, for both cases, can be seen in Fig. 8. High power applications usually leads to possible series and parallel connection of available fuses and must be specially sourced from the manufactures [48], [49]. The manufactures must ensure $<10 \%$ mismatch between different fuses and proper working under normal and fault condition to ensure reliability and protection.

\section{CONCLUSION}

This paper critically analyses the available and proposed technologies for the ship on-board power supplies and the practices of the state of the art MVAC electrical distribution systems in ships. Different technologies are proposed as supply technologies, in literature, for the future MVDC supplies, e.g., high speed gas turbines, high speed PMSGs, and active rectifiers and MMCs for ac/dc conversion. These technologies have their benefits but they are either not commercially available or are too expensive for marine applications. In commercial drives, multipulse rectifiers are connected to multi-secondary transformers to produce high power quality on the dc-side. As transformers are set to be omitted from the emerging MVDC systems, 3-phase generation can be replaced with N-phase generation, which will provide the benefit of using the multi-pulse rectifiers to have high quality de supply.

As illustration, two designs of multi-phase multi-pulse 
MVDC supplies, driven by medium speed ICEs (operated in DAC mode) are presented and discussed. These supplies are: i) a 2 x 3-phase generator interfaced with parallel 12-pulse rectifier for a $18 \mathrm{MW}, 5 \mathrm{kV}$ dc distribution, and ii) a 3 x 3-phase generator interfaced with series 18 -pulse rectifier for a $27 \mathrm{MW}$, $15 \mathrm{kV}$ dc distribution. To realise these supplies, a 6-pulse DRU sub-module is designed with commercially available diodes, which can withstand thermal load under normal operation. Additionally, these two rectifiers are analysed under different fault scenarios and it is shown that they can handle the high surge currents. However, it is also seen that if the faults are not cleared in a few milliseconds, the rectifiers will fail due to every high fault energy. To protect these rectifiers, special purposes fast fuses could be used, placed on the ac-side, to clear these faults.

Additionally, the two notional systems presented here highlight the benefits of multi-phase multi-pulse dc supply. It can be observed that parallel connection of rectifiers improves not only the dc-side supply but also adds redundancy and fault tolerance to the system. Series connection of rectifiers also improves the dc side supply and adds the flexibility in choosing voltage class of generators and motors. The choice of parallel or series rectifiers depends on the system designers, the requirements of the system and the availability of the required equipment.

\section{REFERENCES}

[1] IEEE Recommended Practice for $1 \mathrm{kV}$ to $35 \mathrm{kV}$ Medium-Voltage DC Power Systems on Ships, IEEE Standard 1709, 2010.

[2] J. F. Hansen, J. O. Lindtjøn, and K. Vanska, "Onboard dc grid for enhanced dp operation in ships," in Dynamic Positioning Conf., 2011.

[3] N. H. Doerry and K. McCoy, "Next generation integrated power system: NGIPS technology development roadmap,” Tech. Rep., 2007.

[4] Siemens Marine and Shipbuilding. (2016). Bluedrive plusc - makes vessels safer, more profitable and environmentally friendly. [Online]. Available: https://www.rolls-royce.com/ /media/Files/R/Rolls-Royce/ documents/customers/marine/rr-b-ferry-0317-sp.pdf.

[5] A. K. Adnanes, "Maritime electrical installations and diesel electric propulsion," Tech. Rep., 2003.

[6] B. Zahedi, L. E. Norum, and K. B. Ludvigsen, "Optimization of fuel consumption in shipboard power systems," in 39th Annu. Conf. of the IEEE Industrial Electronics Soc., Nov. 2013, pp. 1124-1129.

[7] A. Tessarolo, S. Castellan, R. Menis, and G. Sulligoi, "Electric generation technologies for all-electric ships with medium-voltage dc power distribution systems," in IEEE Electric Ship Technologies Symp. (ESTS), Apr. 2013, pp. 275-281.

[8] G. Sulligoi, A. Tessarolo, V. Benucci, M. Baret, A. Rebora, and A. Taffone, "Modeling, simulation, and experimental validation of a generation system for medium-voltage dc integrated power systems," IEEE Trans. Ind. Applicat., vol. 46, no. 4, pp. 1304-1310, Jul. 2010.

[9] A. Tessarolo, "Experimental performance assessent of multiphase alternators supplying multiple ac/dc power converters," in 5th IET Int. Conf. on Power Electron., Machines and Drives (PEMD 2010), IET, 2010, pp. 1-6.

[10] T. A. Baragona, P. E. Jordan, and B. A. S. PE, "A breaker-less, medium voltage DC architecture," in ASNE Electric Machines Tech. Symp. (EMTS), 2014, pp. 28-29.

[11] Y. Luo, S. Srivastava, M. Andrus, and D. Cartes, "Application of distubance metrics for reducing impacts of energy storage charging in an mvdc based ips," in IEEE Electric Ship Tech. Symp. (ESTS), Apr. 2013, pp. 287-291.

[12] Y. Luo, C. Wang, L. Tan, G. Liao, M. Zhou, D. Cartes, and W. Liu, "Application of generalized predictive control for charging super capacitors in microgrid power systems under input constraints," in IEEE Int. Conf. on Cyber Technology in Automation, Control, and Intelli- gent Systems (CYBER), Jun. 2015, pp. 1708-1713.

[13] J. M. Meyer and A. Rufer, "A DC hybrid circuit breaker with ultra-fast contact opening and integrated gate-commutated thyristors (IGCTs)," IEEE Trans. Power Del., vol. 21, no. 2, pp. 646-651, Apr. 2006.

[14] K. A. Corzine and R. W. Ashton, "Structure and analysis of the zsource mvdc breaker," in IEEE Electric Ship Tech. Symp. (ESTS), Apr. 2011, pp. 334-338.

[15] H. Mirzaee, S. Bhattachary, and S. Bala, "Design issues in a mediumvoltage dc amplifier with a multi-pulse thyristor bridge front-end," in IEEE Energy Conversion Congr. and Expo. (ECCE), Sep. 2012, pp. 603-609.

[16] D. Dong, Y. Pan, R. Lai, X. Wu, and K. Weeber, "Active fault- current foldback control in thyristor rectifier for de shipboard electrical system," IEEE J. Emerg. Sel. Topics Power Electron., vol. 5, no. 1, pp. 203-212, Mar. 2017.

[17] S. O. Settemsdal, E. Haugan, K. Aagesen, and B. Zahedi, "New enhanced safety power plant solution for dp vessels operated in closed ring configuration," in Dynamic Positioning Conf., Oct. 2014.

[18] U. Javaid, F. D. Freijedo, D. Dujic, and W. van der Merwe, "Marine mvdc multi-phase multi-pulse supply," in 43rd Аnпи. Conf. of the IEEE Industrial Electronics Soc. (IECON), Nov. 2017, pp. 6807-6812.

[19] U. Javaid, D. Dujic, and W. van der Merwe, "Mvdc marine electrical distribution: Are we ready?" in 41st Annu. Conf. of the IEEE Industrial Electronics Soc. (IECON), Nov. 2015, pp. 000 823-00 828.

[20] S. Kanerva, P. Pohjanheimo, and M. Kajava, "Dynamic AC concept for variable speed power generation," ABB Marine and Ports, Helsinki, Finland, Tech. Rep., 2016.

[21] S. Salomon, G. Avigad, R. C. Purshouse, and P. J. Fleming, "Gearbox design for uncertain load requirements using active robust optimization," Engineering Optimization, vol. 48, no. 4, pp. 652-671, 2016.

[22] Wartsila, Wartsila generating sets, 2017.

[23] MAN Diesel and Turbo, Marine engine imo tier ii and tier iii program, 2nd ed. 2016.

[24] GE Power Conversion, Ge showcases innovative and efficient solutions for the maritime industry at smm, 2014.

[25] Bergen Engines AS, Bergen engines products and applications.

[26] GE Aviation, Commercial gas turbines.

[27] Rolls Royce, Gas turbines.

[28] L. Mahon, Diesel generator handbook. Newnes, 1992.

[29] S. Z. Vijlee, A. Ouroua, L. N. Domaschk, and J. H. Beno, "Directlycoupled gas turbine permanent magnet generator sets for prime power generation on board electric ships," in IEEE Electric Ship Technologies Symp. (ESTS), May 2007, pp. 340-347.

[30] ABB, Marine generators - Proven generators for reliable power on board.

[31] Siemens, Siemens electric machines s.r.o., factory drasov, 2010.

[32] GE Power Conversion, Generators - 2500 to $80000 \mathrm{kVA}$ - up to 22000 kVA, 2014.

[33] D. M. Saban, C. Bailey, D. Gonzalez-Lopez, and L. Luca, "Experimental evaluation of a high-speed permanent-magnet machine," in 55th IEEE Petroleum and Chemical Ind. Tech. Conf., Sep. 2008, pp. $1-9$.

[34] Z. W. Vilar and R. A. Dougal, "Load following ability of a ship generating plant comprising a mixed set of high-speed and synchronous turbo-generators," in IEEE Electric Ship Tech. Symp., May 2007, pp. 335-339.

[35] G. Sulligoi, A. Tessarolo, V. Benucci, A. M. Trapani, M. Baret, and F. Luise, "Design, implementation and testing of a shipboard mediumvoltage dc generation system based on a ultra-high speed 12-phase alternator," in IEEE Electric Ship Tech. Symp. (ESTS), Apr. 2011, pp. 388-395.

[36] Power transformers - Part 11: Dry-type transformers. IEC 6007611:2004.

[37] D. Dujic, J. Wahlstroem, J. A. M. Sosa, and D. Fritz, "Modular medium voltage drive for demanding applications," in Int. Power Electron. Conf. (IPEC-Hiroshima - ECCE ASIA), May 2014, pp. 3476-3481.

[38] U. Javaid, F. D. Freijedo, D. Dujic, and W. van der Merwe, "Dynamic assessment of source-load interactions in marine mvdc distribution," IEEE Trans. Ind. Electron., vol. 64, no. 6, pp. 4372-4381, Jun. 2017.

[39] J. Wahlstroem, D. Dujic, M. A. Luescher, and S. Reist, "High power 
igct based multilevel inverter," in Proc. of Int. Exhibition and Conf. for Power Electronics, Intelligent Motion, Renewable Energy and Energy Management (PCIM), May 2014, pp. 1-6.

[40] N. Chaudhuri, B. Chaudhuri, R. Majumder, and A. Yazdani, Multiterminal direct-current grids: Modeling, analysis, and control. John Wiley \& Sons, 2014

[41] A. Christe and D. Dujic, "Galvanically isolated modular converter," IET Power Electron., vol. 9, no. 12, pp. 2318-2328, 2016.

[42] R. Mo, Q. Ye, and H. Li, "Dc impedance modeling and stability analysis of modular multilevel converter for mvdc application," in IEEE Energy Conversion Congr. and Expo. (ECCE), Sep. 2016.

[43] U. Javaid, A. Christe, F. D. Freijedo, and D. Dujic, "Interactions between bandwidth limited cpls and mmc based mvdc supply," in IEEE Energy Conversion Congr. and Expo. (ECCE), Oct. 2017, pp. 26792685.

[44] ABB drives, "Technical guide No. 6 guide to harmonics with AC drives," ABB, Tech. Rep., 2015.

[45] ABB, Application Note 5SYA 2029-03: High power rectifier diodes, 2013.

[46] - - Application Note 5SYA 2051: Voltage ratings of high power semiconductors, 2013.

[47] M. H. Rashid, Power electronics handbook: devices, circuits and applications. Academic press, 2010.

[48] Ferraz Shawmut, Mersen, Special Purpose Fuses, 2013.

[49] Eaton, Application Guide 10507: Protecting semiconductors with high speed fuses, 2016.

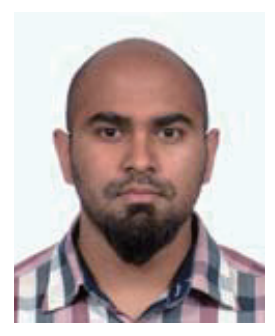

Uzair Javaid was born in Lahore, Pakistan in 1989. He received his B.Sc. in Electrical Engineering. in 2010, from University of Engineering and Technology, Lahore. He obtained his M.Sc. in Energy Management and Sustainability from École Polytechnique Fédérale de Lausanne (EPFL) in 2013. Since April 2014, he is a doctoral assistant in Power Electronics Laboratory in EPFL. His research interests include MVDC distribution systems, power electronic system modeling and design and electri-

cal machine drives.

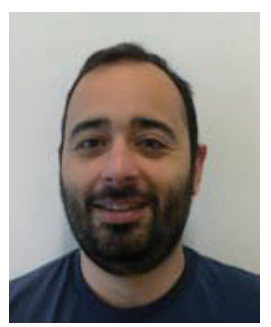

Francisco D. Freijedo received the M.Sc. degree in physics from the University Santiago de Compostela, Santiago de Compostela, Spain, in 2002 and the Ph.D. degree in electrical engineering from the University of Vigo, Vigo, Spain, in 2009. From 2005 to 2011, he was a Lecturer in the Department of Electronics Technology, University of Vigo. From 2011 to 2014, he worked with Games Innovation and Technology as a Power Electronics control Engineer for renewable energy applications. From 2014 to 2016, he was a Postdoctoral Researcher in the Department of Energy Technology, Aalborg University. Since 2016, he is a Scientific Collaborator of the Power Electronics Laboratory, École Polytechnique Fédérale de Lausanne. His main research interests include power conversion technologies.

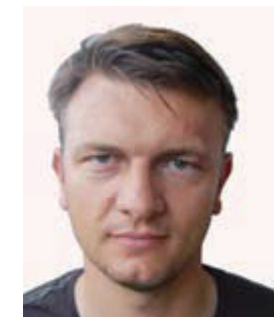

Drazen Dujic received the Dipl. -Ing. and M.Sc. degrees from the University of Novi Sad, Novi Sad, Serbia, in 2002 and 2005, respectively, and the Ph.D. degree from the Liverpool John Moores University, Liverpool, U.K., in 2008, all in electrical engineering. From 2002 to 2006, he was with the Department of Electrical Engineering, University of Novi Sad as a Research assistant, and from 2006 to 2009 with Liverpool John Moores University as a Research Associate. From 2009 till 2013, he was with ABB Corporate Research Centre, Switzerland, as a Principal Scientist working on the power electronics projects spanning the range from low-voltage/power SMPS in below kilowatt range to medium voltage high-power converters in a megawatt range. During 2010-2011, he was a member of a project team responsible for the development of the worlds first power electronic traction transformer (PETT) successfully commissioned on the locomotive. From 2013 till 2014, he was with ABB Medium Voltage Drives, Turgi, Switzerland, as R\&D Platform Manager, responsible for ABB's largest IGCT based medium voltage drive ACS6000. He is currently with École Polytechnique Fédérale de Lausanne EPFL, Lausanne, Switzerland, as an Assistant Professor and the Director of the Power Electronics Laboratory. His current research interests include the areas of design and control of advanced high-power electronics systems and high performance drives. He has authored or coauthored more than 80 scientific publications and has filed eleven patents. He is an Associate Editor for IEEE Transactions on Industrial Electronics, IEEE Transaction on Power Electronics and IET Electric Power Applications. He has received the First Prize Paper Award by the Electric Machines Committee of the IEEE Industrial Electronics Society at IECON-2007. In 2014 he has received the Isao Takahashi Power Electronics Award for outstanding achievement in power electronics.

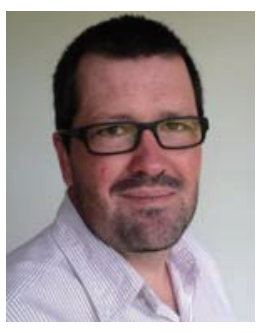

Wim van der Merwe received the B.Eng., M.Sc. (Eng), and Ph.D. degrees, in electrical engineering from the University of Stellenbosch Stellenbosch, South africa, in 2000, 2006, and 2011, respectively. He joined ABB Corporate Research 2012 and is currently R\&D Platform Manager of he ACS6000 MV-drive at ABB Medium Voltage Drives in Turgi, Switzerland. His research interests include multilevel and medium-voltage power electronic converters and the modeling of complex power electronic systems. 